In: Henri Schoenmakers, Stefan Bläske, Kay Kirchmann, Jens Ruchatz (Hg.),

Theater und Medien / Theatre and the Media, Bielefeld: transcript, 2015, S. 161-169

\title{
Medium, Theater und Film bei Beckett
}

\author{
FRANZISKA SICK
}

I.

Becketts Umgang mit Theater und Film ist avantgardistische Medienkunst. Unter Medienkunst fasse ich hierbei nicht (oder nicht primär) den Einsatz neuer Medien, sondern Aspekte wie Medienreflexivität und intermediale Strukturanalogien bis hin zur Mediendurchwirktheit von Subjektivität. Es geht deshalb nicht darum, Beckett als Hörspielautor oder Vorläufer der Videokunst vorzustellen, sondern um seine medienreflexiven Theaterstücke aus den frühen 1960er Jahren und um FILM.

Der Avantgardismus Becketts lässt sich mit am genauesten im Kontrast zur Ästhetik des Nouveau Roman bestimmen. Auch die Autoren des Nouveau Roman reflektieren mediale Aspekte von Kunst, doch geht es ihnen dabei primär um die Erneuerung des Romans. Selbst Alain Robbe-Grillet, der sich programmatisch auf den Film bezieht und damit eine vergleichsweise moderne Position vertritt, hebt am Film zuvörderst seine Bildlichkeit hervor und rezipiert ihn damit im Grunde medienkonservativ. Denn einerseits gibt es Bildlichkeit und Beschreibung im Roman bereits vor der Erfindung des Films, und andererseits greift Robbe-Grillet (1963: 19) das eigentliche Novum des Tonfilms, dass bewegte Bilder mit Ton und Sprache unterlegt sind, nicht auf. Nathalie Sarraute weist die Orientierung am Film ganz zurïck. Ihr zufolge ist der Nouveau Roman dialogisch, und sie setzt ihn deshalb in Beziehung zum Theater (1964: 1591). Beide - Robbe-Grillet wie Sarraute - verkürzen in ihrer Apologie des Romans das Referenzmedium, auf das sie sich beziehen: Robbe-Grillet blendet aus dem Film die Bewegung der Bilder und den Dialog aus, Sarraute abstrahiert in ihrer Lesart des Theaters von Gestik und Bühnenbild.

Derlei Abstraktionen finden sich durchaus auch bei Beckett - und dies sogar in größerem Umfang. Sie haben bei ihm eben deshalb jedoch einen anderen Stellenwert. SPIEL OHNE WORTE kommt, wie bereits der Titel annonciert, ohne das aus, was man gängiger Weise von einem Drama erwartet: Worte. Ohne dramatische Wechselrede reduziert sich das Theater auf reine Pantomime. In QUADRAT verzichtet Beckett selbst noch auf den pantomimischen Ausdruck und gestaltet eine Choreographie rein räumlicher Bewegungen. Andererseits schreibt Beckett Stücke, die tendenziell nur aus Sprache bestehen - aus einer rein >dialogischen < Spielsituation wie in SPIEL oder aus einem reinen Monolog wie in NOT I.

Im Grundansatz stellt Beckett damit die frühe technische Medienentwicklung nach. Technikbedingt war diese von Abstraktionen geprägt: Starre Bilder in der Photographie, bewegte, aber stumme Bilder im Film, Sprache ohne Bild im Radio und auf Tonträgern. In unterschiedlicher Weise ist Becketts Theater- und Filmschaffen von diesen Disjunktionen - ohne Worte, ohne Bewegung, mit Bewegung, mit Sprache - durchsetzt, für die er immer neue Spielanordnungen erfindet. Dem technischen Erfindungsreichtum der Medien setzt er einen anderen, primär ästhetisch motivierten entgegen. Im Grundansatz folgt Beckett damit den Surrealisten 
(man denke an Marcel Duchamp, Max Ernst oder Yves Tanguy), die auf den maschinentechnischen Erfindungsreichtum des frühen 20. Jahrhunderts mit der Erfindung abstruser Maschinen antworteten. Beckett überträgt dieses Modell in eine Kommunikations- und Medienästhetik. Seine Modernität besteht mit in diesem Paradigmenwechsel, sein Avantgardismus im Versuch, stets grundlegend neue Spielarten zu erfinden.

Wie sehr Beckett selbst ästhetisch nicht überformte, also bloß technischmediale Beschränkungen als ästhetische Figur wahrnimmt, zeigt seine Vorliebe für den Stumm- gegenüber dem Tonfilm. Technikgeschichtlich läutet der Tonfilm mit der Zusammenführung von Bild- und Tonspur das multimediale Zeitalter ein (Kittler: 254). Beckett verweigert sich dieser Entwicklung. Mit FILM legt er einen Tonfilm vor, der wie ein Stummfilm inszeniert ist. Kein Wort wird in diesem Film gewechselt - mit einer Ausnahme: Als ein Angerempelter aufbrausen will, bringt seine Begleiterin ihn mit einem »Psst « zum Schweigen - den Angerempelten im Film, aber auch den Tonfilm. Trotz dieser Zurückweisung des Tons in FILM hat Beckett sich nicht gescheut, SPIEL, das reiner >Dialog < unter Ausblendung jeglicher Mimik und Gestik ist, zu verfilmen. Beides ist für ihn im Tonfilm erlaubt - der Ton und das bewegte Bild -, nur nicht beides zugleich, zumindest tendenziell.

Medienkunst in Theater und Film ist deshalb bei Beckett nicht primär in einer unmittelbaren Weise zu verstehen, so als würde sie in vollem Umfang die Möglichkeiten eines Mediums ausloten. Vielmehr ist sie als eine Inszenierung unterschiedlicher (quasi) medialer Konstellationen zu bestimmen.

Mediale Basis (in einem engen Sinne des Wortes) solcher Inszenierungen sind Medien, die multimediale Möglichkeiten bieten wie der Film oder das Theater. Dass Beckett sich mit dem Theater eines alten Mediums bedient, tut seinem Avantgardismus keinen Abbruch. Denn entscheidend ist nicht, welches Medium man verwendet, sondern wie medienreflexiv man es einsetzt (Boenisch: 453f). In dem multimedialen Raum, als der sich das Theater zumindest im Rückblick erweist (wie der Tonfilm kann das Theater bewegte, sprechende Körper darstellen), führt Beckett Spielarten medialer Beschränkung vor. Gerade weil das alte Medium vielseitiger ist als die neuen, lässt sich in ihm deren mediale Beschränkung abbilden, aber auch um weitere, erfundene mediale Konstellationen ergänzen.

Nun gehören Beschränkungen wie der Verzicht aufs Wort in Pantomime und Tanztheater zum Grundbestand der Theaterkultur, und es stellt sich deshalb die Frage, ob man die Vielfalt Beckettscher Spielsituationen nicht besser gattungsästhetisch deuten sollte. Um die Frage zu klären, ist wenigstens ansatzweise eine Abgrenzung beider Begriffe (Medium und Gattung) erforderlich: Medien sind Darstellungsräume, die immer auch Darstellungsbeschränkungen beinhalten. So kann ein Radio keine Bilder senden, ein Stummfilm keinen Ton. Gattungen basieren auf Medien und schränken den medialen Darstellungsraum durch Gattungskonventionen zusätzlich ein - in der Pantomime kommt Sprache nicht vor. Unter rein formalen Gesichtspunkten könnte man deshalb die Beckettschen Abstraktionen durchaus auch gattungsästhetisch deuten.

Dennoch besteht ein Unterschied: Man nimmt in einer Ballettaufführung nicht wahr, dass die Tänzer nicht reden, sondern sieht die anderen Ausdrucksformen des Körpers, die durch den Verzicht auf Rede möglich sind. Anders verhält es sich bei Beckett. Die Einschränkung der Spielmöglichkeit erscheint bei ihm wie ein Zwang oder wie eine Grenze, die man nicht überschreiten kann. Das »Psst « in FILM ist auch vor diesem Hintergrund zu deuten. In SPIEL und NOT I sind die Figuren wie zementiert, in QUADRAT umkreisen sie einen Punkt, von dem sie wie von einem 
Magnetfeld angezogen und abgestoßen werden. Becketts Figuren sind wie eingesperrt in eine Spielsituation, der sie nicht entkommen können, und die sie in

ihren Bewegungs- und Äußerungsmöglichkeiten einschränkt. Deshalb erscheinen die Beckettschen Inszenierungen wie Medien.

Ein weiterer Aspekt kommt hinzu. Da bei Beckett die formale Beschränkung der Spielsituation stets reflexiv mit zur Darstellung kommt, können seine Stücke nicht gattungsbildend wirken. Unterlegte man SPIEL einen anderen Text, entstünde kein neues Stück, sondern allenfalls eine Variante des bestehenden.

\section{II.}

In SPIEL stecken drei Figuren in je einer Urne. Ihr Gesicht ist dem Publikum zugewandt. Die Bühne ist dunkel. Die Figuren werden abwechselnd und in schnellem Rhythmus mit einem Spot so angeleuchtet, dass man nur jeweils die angeleuchtete Figur sieht. Wenn eine Figur angeleuchtet wird, fängt sie zu sprechen an, und sie verstummt abrupt, wenn eine andere Figur angeleuchtet wird.

Man hat vielfach darauf hingewiesen, dass der schnelle Lichtwechsel an die Schnitttechnik des Films erinnert. Jedoch lässt sich SPIEL nicht auf solche Oberflächeneffekte und bloße Medienzitate reduzieren. SPIEL definiert einen eigenen intermedialen Raum, in dem Momente der Erzählung, des Films und des Theaters zu einem neuen Darstellungsraum verschmelzen.

Als ein Teil dieser medialen Konfiguration ist die Textform von SPIEL - SPIEL als Lesedrama oder aber auch als Erzählmontage - zu bestimmen. Das Stück besteht aus kurzen Dialogsequenzen. Wenn man diese entgegen der Textanordnung so liest, dass man die Rede einer Figur verfolgt und hierbei jeweils die Reden der beiden anderen überspringt, erhält man drei vergleichsweise kohärente Geschichten. Jede der Figuren stellt ihre Sicht auf das Ehedrama und Dreiecksverhältnis dar, das zwischen den drei Akteuren besteht. Entgegen seinem Titel, der auf das (Schau-)Spiel hindeutet, besteht SPIEL in der textuellen Grundanlage aus drei Erzählungen. Beckett zerschneidet diese Erzählmonologe und überlagert sie zeitlich. Das würde zu einer nicht weiter irritierenden Umsetzung von Erzählperspektiven in das Theatermedium führen, wenn Beckett nicht ein wesentliches Detail äußerst ungewöhnlich gestalten würde: Er verkürzt die einzelnen Erzählausschnitte so stark (sie umfassen zumeist kaum mehr als 1-3 Zeilen), dass sich kein eigenständiger Erzählfokus herausbilden kann. Man kann sich diese mediale Funktionsweise des Stücks in Analogie zum Film vergegenwärtigen. Bekanntlich basiert der Film darauf, dass das menschliche Auge ab etwa 16 Bilder pro Sekunde diese nicht mehr als einzelne Bilder wahrnehmen kann. Da Filme gewöhnlich mit 24/25 Bildern pro Sekunde produziert und abgespielt werden, nehmen wir die Einzelbilder nur als Bewegungsfluss wahr. Würde man einen Film langsamer abspielen, würden die Bilder ruckeln. Beckett überträgt diesen Effekt in eine andere mediale Konstellation: So wie ein Film, der mit der falschen Geschwindigkeit abgespielt wird, ruckelt, so ruckelt in SPIEL die erzählerische Fokalisation (zum Begriff der Fokalisation: Genette: 48-52). Das Stück ist in dieser Hinsicht auch als Travestie des Films anzusehen.

Wie sehr SPIEL an Filmästhetik angelehnt ist, zeigt sich nicht zuletzt an seiner räumlichen Anordnung. So wie im Kino eine Lichtquelle, die sich im Rücken der Zuschauer befindet, ein Geschehen projiziert, so in der Grundkonstellation auch bei Beckett. Als mediale Anordnungen sind auch solche Lichtverteilungen zu verste- 
hen, denn sie erschaffen - unabhängig von den architektonischen Gegebenheiten, die weitgehend stabil bleiben - allererst den (Theater-)Raum und damit das, worin etwas erscheinen kann, also das Medium (vgl. Sick: 47f). Ich kann an dieser Stelle die mediale Relevanz des Lichts im Theaterraum nur stichwortartig skizzieren: Das Sprechtheater im 17. Jahrhundert ist dadurch geprägt, dass die Schauspieler an die mit Kerzen beleuchtete Rampe vortreten müssen, um besser sichtbar zu sein. Das Licht fixiert sie an die Rampe und lässt ein Spiel in der Tiefe des Bühnenraums nicht zu. Noch die so genannte vierte Wand ist entgegen der architektonischen Metapher nichts weiter als ein Beleuchtungseffekt: Man dunkelt den Zuschauerraum ab und markiert damit, dass die Welt auf der Bühne eine eigene ist. Mit dem Kino entsteht eine weitere Beleuchtungsarchitektur und mit ihr ein neuer Fiktionsraum. Ohne dass sich der Aufführungsraum grundlegend verändert, besitzt die »Bühne« im Kino nurmehr den Stellenwert einer Projektionsfläche. Das zeigt sich am genauesten dort, wo sich im Kino atavistischer Weise ein Vorhang öffnet. Denn anders als im Theater gibt der Vorhang im Kino den Blick nicht auf eine andere Welt (den Bühnenraum), sondern nur auf eine weiße Wand frei.

Becketts SPIEL übernimmt vom Kino den Projektor und von der Bühne den Fiktionsraum. In dieser intermedialen Kombination entsteht gegenüber den beiden Ausgangsmedien ein neuer Aufführungsraum, der einigermaßen genau die Schwebe zwischen (Licht-)Projektion und (materieller) Aufführung einhält. Dennoch ist SPIEL immer noch Theater, wenn auch in Schrumpfform. In der intermedialen Konfrontation von Kino und Theater zeigt sich in nachgerade medienanalytischer Weise der irreduzible Rest, der das Theater vom Kino unterscheidet: Theater findet dort statt, wo Menschen sich auf einer Bühne befinden. Der Umstand, dass die Akteure von SPIEL in Urnen stecken, unterstreicht dies. Es geht mehr um die Existenz der Figur als um ihre lebendige Gestik.

So formal diese Spielanordnung ist, so groß ist doch zugleich ihre semantische Wertigkeit. Das lässt sich anhand eines Gedankenexperiments zeigen. Da SPIEL weitgehend auf Lichtprojektionen basiert, könnte man die Schauspieler auf der Bühne durchaus auch durch gefilmte Schauspieler ersetzen. Innerhalb der Aufführung wäre diese Manipulation kaum wahrnehmbar. Dennoch würde eine solche Aufführung das Stück entscheidend verändern: Die filmische Projektion würde das Bild von Figuren zeigen, die sprechen. SPIEL zeigt demgegenüber Figuren, die sprechen, wenn sie angeleuchtet werden. Obwohl das Erscheinungsbild sich für den Zuschauer nicht verändert, nehmen wir beide Situationen unterschiedlich wahr. Entgegen der Fiktion von der vierten Wand verstehen wir, zumindest in einem solchen Grenzfall, das Geschehen auf der Bühne immer auch von seinem Aufführungsrahmen her. Man hat im 20. Jahrhundert die vierte Wand durch vergleichsweise grobschlächtige Aktionen wie die PUBLIKUMSBESCHIMPFUNG einzureißen versucht - Beckett dekonstruiert sie ungleich subtiler: durch intermediale Reflexion.

Wenn eine Figur angeleuchtet wird, spricht sie. Das Licht in Becketts SPIEL ist wie ein Befehl: »Du stehst im (Rampen-)Licht, sprich!« Man kann sich unterschiedliche Szenarien vorstellen, die diese Grundsituation verbildlichen: einen Erzähler, der die Figuren zum Sprechen bringt, oder - an den Befehlscharakter des Lichts anschließend - eine Verhörsituation mit einer Lampe. Indessen sind dies Hilfsvorstellungen, die der Abstraktheit von SPIEL nicht gerecht werden. Der einzige Inhalt, der in diesem Stück gesetzt ist, besagt, dass das Licht befiehlt. Dies allerdings äußerst präzise. Das zeigt kontrastiv die Verfilmung des Stücks, in der, anders als im Bühnenstück, der Sprecherwechsel nicht als harter Schnitt gesetzt ist. 
In der Verfilmung von SPIEL schwankt die Kamera, wenn sie einen neuen Sprecher fokussiert, so als müsste sie ihn erst suchend umkreisen, um erst nach einiger Zeit zu einer ruhigen Bildeinstellung zu gelangen. Während das Licht befiehlt, sucht die Kamera. Man sieht an dieser Stelle, in wie präziser Weise Beckett Medien mit menschlichen Verhaltensweisen in Verbindung bringt.

Dass in SPIEL die Aufführung oder das Medium selbst zur Aufführung kommt, lässt sich theaterästhetisch einigermaßen genau anhand einer weiteren Auffälligkeit von SPIEL belegen. Typischer Weise entspringt die Handlung auf dem Theater der Wechselrede. Ein Wort gibt das andere oder fordert zumindest zur Antwort und Stellungnahme auf. Dem Vorhang kommt dabei lediglich eine rahmende Funktion zu. Er öffnet den Sprechraum und schließt ihn, aber er greift nicht in den Dialograum ein. Becketts SPIEL besitzt eine gänzlich andere Grundgeometrie. In SPIEL entspringt der Wechsel der Rede nicht länger einem dramatisch fundierten Redewechsel, sondern einem Beleuchtungswechsel. Während im Falle des traditionellen dramatischen Redewechsels es dem Gegenspieler zufällt, seinem Kontrahenten das Wort abzuschneiden, verlegt Beckett diese urdramatische Konstellation auf die Ebene der Inszenierung. Obwohl das Licht reines Medium ist, ist es die eigentliche dramatis personae des Stückes. Das Medien-Drama von SPIEL findet, wenn nicht hinter der Bühne, so doch im Rücken der Zuschauer statt.

\section{III.}

Wir haben gesehen, dass bei Beckett Medien wie das Licht oder die Kamera Aktcharakter besitzen. FILM setzt diese mediale Semantik fort - und verschärft sie zugleich. Während in der Verfilmung von SPIEL die Kamera nur beim Schnittwechsel in einer suchenden Bewegung ihren Gegenstand fokussiert, nimmt sie in FILM buchstäblich eine Verfolgungsjagd auf.

FILM setzt ein mit einer Kamera, die eine Mauer abtastet. Bald findet sie ihr gesuchtes Objekt: einen Mann in Rückenansicht. Nach einer Verfolgungsjagd durch die Straßen flüchtet der Mann in ein Zimmer und entfernt in ihm alles, was Augen hat: einen Hund, eine Katze, weitere Okulare verhängt er. Trotzdem ist in dem Zimmer noch eine Sehinstanz verblieben: die der Kamera. Die Schlusseinstellungen machen deutlich, dass dieser verbleibende Rest das Ich selbst ist, nicht als Betrachtetes, sondern als sich Betrachtendes. Das Ich war also in letzter Instanz vor seiner Selbstwahrnehmung auf der Flucht. Eine philosophische Vorbemerkung zu dem Drehbuch, die George Berkeley zitiert und auf René Descartes anspielt, unterstreicht diese Lesart (Beckett 1965: 339): »Die Suche nach dem Nicht-Sein durch Flucht vor der Wahrnehmung anderer scheitert an der Unausbleiblichkeit der Selbstwahrnehmung.«Andererseits entwertet Beckett seine Vorüberlegungen mit dem Hinweis, sie seien bloß als Regieanweisung zu lesen.

Dass FILM trotz dieses philosophischen Gehalts primär medienreflexiv zu verstehen ist, zeigt nachgerade programmatisch der Titel: FILM ist nicht ein Film, sondern der Film, es gibt keinen anderen. Denn FILM zeigt, was Film ist: eine so suchende wie zugleich bewegliche Kamera. Als medienreflexiv ist ferner $\mathrm{zu}$ werten, dass in FILM die Kamera nicht nur etwas zeigt, sondern selbst als Akteur auftritt. Darüber hinaus fällt auf, dass FILM in Anlehnung an den Stummfilm eine Vielzahl clownesker Szenen enthält. Es stellt sich deshalb die Frage, ob FILM philosophisch und ernst (Breuer 1995) oder medienästhetisch und heiter zu interpretieren ist. Gegen eine rein philosophische Ausdeutung von FILM ist einzuwen- 
den, dass die Selbstdeutung des Subjekts immer schon mediengebunden ist. Bereits die Metapher der Reflexion im Begriff der Selbstreflexion deutet darauf hin: Sie ist am Spiegel, also an einem Medium, abgelesen.

Der Glaube an eine adäquate Selbstwahrnehmung löst sich wohl mit am radikalsten mit der Photographie auf. Als die Kamera beweglich wurde, verfielen Photographen auf die Idee, am eigenen Körper herunter zu photographieren. Mit dem dritten, beweglichen Auge der Kamera lässt sich zeigen, was in der alltäglichen Selbstwahrnehmung tatsächlich zu sehen ist: ein fragmentarischer Körper.

Auf den imaginären Charakter der Ich-Bildung hat schon früh Melanie Klein (57-72) hingewiesen - ihre Theorie vom zerstückelten Körper erinnert an die Selbstbilder der frühen Photographen. Später dekonstruiert Jacques Lacan (89-97) mit Verweis auf den imaginären Charakter des Spiegelbildes Kants transzendentale Einheit der Apperzeption. So hat, wenn auch auf Umwegen, die bewegliche Kamera das Vertrauen in die Selbstreflexion erschüttert und abgelöst. Wir können Selbstreflexion nicht länger nach dem Modell des Spiegels, sondern nur noch nach dem Modell der Kamera denken, also als vergebliches Unterfangen.

Genau dieses Modell einer primär technikinduzierten Selbstwahrnehmung und -reflexion führt Beckett in FILM vor. Er greift die Selbstbilder der beweglichen Kamera auf und adaptiert sie an den Film. Und so wird aus den torsohaften Körperbildern, die bewegliche Photoapparate liefern können, das Drama einer Flucht vor sich, das die Filmkamera abbilden kann.

$\mathrm{Zu}$ fragen ist also nicht, ob FILM das Subjekt oder das Medium der beweglichen Kamera darstellt (Schwab 1996), denn beides lässt sich kaum voneinander trennen. Und ebenso wenig ist zu fragen, ob die vorgestellte Auffassung vom Subjekt wahr ist. Sie ist nichts weiter als eine mediale Spielanordnung, vor deren Hintergrund wir uns selbst verstehen. Beckett reflektiert diese Bezüge im Titel FILM, der auf das Medium verweist, und in der Regieanweisung, die die Wahrheit zugunsten der Inszenierung kassiert.

\section{IV.}

So wie FILM ein Selbstverhältnis medial abbildet und zugleich dissoziiert, so auch DAS LETZTE BAND. Das Stück handelt von einem alten Mann, der seine Lebenserinnerungen auf Tonband gesprochen hat. Bevor er weitere Erinnerungen und Selbsteinschätzungen aufnimmt, hört er das Band ab. Er spult dabei das Tonband häufig vor und zurück. Hieraus entsteht folgende verschachtelte Situation: Wir hören vom Tonband den jungen Krapp, wir hören vom Tonband Krapp als Mann mittleren Alters, der über sich als jungen Mann, aber auch über seine gegenwärtige Situation spricht. Und wir hören und sehen auf der Bühne den alten Krapp, der das Band abhört und die Ansichten des jungen und des in die Jahre gekommenen Krapp gestisch, aber auch verbal kommentiert.

In DAS LETZTE BAND zerlegt Beckett das Gedächtnis in nachgerade analytischer Weise in seine Teilfunktionen: Die aktive Funktion der Erinnerung und die passive Funktion der Speicherung treten auseinander und differenzieren sich weiter - in ein System von Registern, Schachteln und Spulen hier und in ein Hervorkramen, Tonband-Einfädeln, Vor- und Zurückspulen dort.

So wie sich in FILM das Subjekt in eine technische Repräsentanz und in einen Subjektrest spaltet, so auch in DAS LETZTE BAND. Obwohl die Aktivitätspotentiale unterschiedlich besetzt sind - in FILM verfolgt die Kamera das leibhaftige Ich, in 
DAS LETZTE BAND kramt das leibhaftige Ich in den medialen Sedimenten des auf Band aufgezeichneten Ich -, ist beiden Konstellationen eine gemeinsame Grundfigur eigen: So sehr Medien den Zugang zu sich allererst eröffnen und objektivieren, so sehr dissoziieren sie das Ich und entfremden es damit von sich. Erst unter dem Auge der Kamera wird deutlich, dass man sich nicht reflexiv selbst wahrnehmen kann, erst unter den Bedingungen des Tonbands zeigt sich in voller, da medialer Schärfe, dass man die eigenen Erinnerungen nicht wahrhaben will. Krapp beschimpft sein eigenes vergangenes Ich und schneidet, das Tonband vor- und zurückspulend, dem jungen Krapp wiederholt das Wort ab.

Indem Beckett einen Aspekt des Subjekts - Selbstwahrnehmung bzw. Erinnerung - analytisch herausgreift und in Medium (Kamera bzw. Tonband) und Subjekt aufspaltet, wird so etwas wie das Drama des Subjekts darstellbar. Ein solches Subjekt-Drama ist erst dort möglich, wo das Subjekt Teilfunktionen von Subjektivität in Medien objektiviert. Nur so kann es auf der Bühne (oder auch im Film) einen geeigneten Widerpart finden und sich in veräußerlichter Weise selbst begegnen. Nur unter dieser Voraussetzung auch kann man auf den Gegenspieler auf der Bühne verzichten und entgegen der Gattungskonvention ein rein monologisches Drama schreiben.

Wie SPIEL lässt sich DAS LETZTE BAND als Inszenierung einer Erzählmontage deuten: So wie in SPIEL die Fokalisation der drei Protagonisten durch das Licht erfolgt, so setzen sich in DAS LETZTE BAND die Erinnerungen des alten Krapp aus Tonbandaufzeichnungen zusammen. Da sich Krapps Erinnerungen vielfältig überlagern, ist ein Vergleich mit Prousts Roman A LA RECHERCHE DU TEMPS PERDU naheliegend (Lommel: 260-264). Was die Aufschreibesituation anbelangt, liegt der Vergleich mit dem Tagebuch jedoch näher. Denn wie ein Tagebuchschreiber zeichnet Krapp zeitnahe Erinnerungen auf. DAS LETZTE BAND ist gleichwohl keinem dieser beiden Bezugsgrößen (Erinnerungsroman und Tagebuch) eindeutig zuzuordnen, da es deren Grunddisjunktion unterläuft. Diese besteht in einer einigermaßen eigenartigen Aufgabenverteilung von deutender Erinnerung und Archivierung der Erinnerung. Während Prousts Ich-Erzähler sein Leben nachträglich zu erinnern und zu deuten versucht, ohne Tagebuch zu führen, hält der Tagebuchschreiber seine Erinnerungen zeitnah fest, ohne sie in einem umfassenden, lebensübergreifenden Sinne zu deuten. DAS LETZTE BAND hebt genau diese Disjunktion auf: Krapp versucht eine Gesamtdeutung seines Lebens auf der Basis aufgezeichneter früherer Erinnerungen. Ironischer Weise misslingt dies, weil frühere Lebensentwürfe mit den aktuellen konfligieren.

Eine vergleichbare Konstellation findet sich in Michel Butors Tagebuchroman L'EMPLOI DU TEMPS, der annähernd zeitgleich mit DAS LETZTE BAND erschienen ist. Butors Tagebuch schreibender Protagonist versucht, mit Hilfe seiner Aufzeichnungen einen Kriminalfall zu lösen. Entgegen der Gattungskonvention des Tagebuchromans und durchaus wie Krapp unterzieht Butors Protagonist seine alten Aufzeichnungen einer wiederholten Relektüre und kommentiert diese. Die Selbstbegegnung mit dem vergangenen Ich ist also nicht primär mediengebunden. Ebenso sehr aber gilt, dass die Möglichkeiten der alten Medien erst nach der Erfindung neuer Medien vollständig ausschöpfbar sind. Erst wenn auf dem Tonband die eigene, vergangene Stimme zu hören ist, verfällt man in der Literatur auf die Idee, einen Tagebuchroman zu schreiben, der von der Vergegenwärtigung des Geschriebenen durch wiederholte Lektüre handelt.

Man kann es auch so ausdrücken: Vor dem Hintergrund neuer medialer Spielmöglichkeiten verlieren die konventionellen Gattungsgrenzen ihre selbstverständ- 
liche Gültigkeit. In SPIEL und DAS LETZTE BAND stellen medieninduzierte Erzählanordnungen die Basis dar (SPIEL beschleunigt den Wechsel der Fokalisation vor dem Hintergrund filmischer Schnitttechnik bereits in der textuellen Anordnung, Krapp kommentiert sein altes Ich). Gleichsam in einem zweiten Schritt überträgt Beckett diese modifizierten Erzählmodelle auf das Theater, um sie dann in einem dritten Schritt mit modernen Medien wie fortgeschrittener Beleuchtungstechnik und Tonband umzusetzen. So verschränkt sind die intermedialen Transformationen zwischen Erzählung und Medium, Erzählung und Theater und dem Einsatz neuer Medien auf dem Theater bei Beckett.

Anders als der große Schweiger Beckett haben die Autoren des Nouveau Roman ihre poetische Praxis vielfach reflektiert. Butors L'EMPLOI DU TEMPS ist strukturgleich mit Becketts DAS LETZTE BAND. Und so stellt sich die Frage, ob man Becketts ästhetisches Verfahren vom Theorieansatz Butors her verstehen kann. Die Antwort auf diese Frage findet sich in einem kleinen Aufsatz, in dem Butor (1964) die alten Medien gegenüber den neuen zu verteidigen sucht, indem er diesen nachrechnet, dass sie so antiquiert sind wie die Buchkultur in ihren Anfängen. Denn so wie Filme und Tonbänder aufgewickelt sind, so waren auch Bücher anfänglich aufgerollt. Über den Fortschritt der Medien mag man sich streiten. Butor stellt meines Erachtens eine grundsätzlichere Frage: Mediale Konstellationen sind zuvörderst von Anordnungsbeziehungen her zu klären, und zu Teilen eröffnen die Anordnungsbeziehungen der technischen neuen Medien allererst den Blick auf die konventionellen und gattungsmäßigen Beschränkungen der alten.

Ich habe Beckett eingangs aufgrund der Analytik seiner Spielsituationen in etwas metaphorischer Weise als Medienkünstler bezeichnet. An dieser Stelle schließt sich die Argumentation: Wenn man bei Beckett >konkrete< Medien zu analysieren versucht, stellt man fest, dass deren Medialität nicht nur in ihrer medialen Dinglichkeit, sondern ebenso sehr in Spielstrukturen und Anordnungsbeziehungen besteht. NOT I gibt zu hören und zeigt eine Stimme: einen sprechenden und beleuchteten Mund und einen Zuhörer. Kein Medium im strengen Sinne des Wortes befindet sich auf der Bühne - und dennoch ist das Stück von derselben Dekomposition des Subjekts geprägt wie DAS LETZTE BAND, an das es in Abwandlungen erinnert, denn auch hier haben wir es mit einem Sprecher und einem Zuhörer zu tun.

\section{Literatur}

Beckett, Samuel (1963): »Spiel«. In: Samuel Beckett (1967): Stücke, S. 311-328.

Beckett, Samuel (1965): »Film«. In: Samuel Beckett (1967): Stücke, S. 337-355.

Beckett, Samuel (1967): Stücke. Kleine Prosa. Auswahl in einem Band. Deutsch von Erika und Elmar Tophoven. Frankfurt/M.: Suhrkamp.

Beckett, Samuel (1973): Not I. London: Faber \& Faber.

Beckett, Samuel (1974): Das letzte Band (1959). Dreisprachig mit Szenenphotos. Frankfurt/M.: Suhrkamp.

Boenisch, Peter M. (2003): »Theater als Medium der Moderne? Zum Verhältnis von Medientechnologie und Bühne im 20. Jahrhundert«. In: Christopher Balme/Erika Fischer-Lichte/Stephan Grätzel (Hg.): Theater als Paradigma der Moderne? Positionen zwischen historischer Avantgarde und Medienzeitalter. Tübingen und Basel: Francke, S. 447-456.

Breuer, Rolf (1995): »Beckett's Film. Philosophical and Poetological Implications«. Germanisch-romanische Monatsschrift, 45, S. 463-467. 
Butor, Michel (1956): L'emploi du temps. Paris: Minuit.

Butor, Michel (1964): »Le livre comme objet«. In: Michel Butor: Essais sur le roman. Paris: Gallimard, S. 130-157.

Genette, Gérard (1983): Nouveau discours du récit. Paris: Seuil.

Kittler, Friedrich (1986): Grammophon Film Typewriter. Berlin: Brinkmann \& Bose.

Klein, Melanie (1930): »Die Bedeutung der Symbolbildung für die Ich-Entwicklung «. In: Internationale Zeitschrift für Psychoanalyse, 16, S. 57-72.

Lacan, Jacques (1949): »Le stade du miroir comme formateur de la fonction du Je«. In: Jacques Lacan (1966): Ecrits I. Paris: Seuil, S. 89-97.

Lommel, Michael (2004): »Synästhesie der Erinnerung: Becketts Krapp's Last Tape«. In: Franziska Sick/Beate Ochsner (Hg.): Medium und Gedächtnis. Von der Überbietung der Grenze(n). Frankfurt/M.: Peter Lang, S. 255-264.

Robbe-Grillet, Alain (1963): Pour un nouveau roman. Paris: Minuit.

Sarraute, Nathalie (1964): »L'Ere du soupçon«. In: Nathalie Sarraute (1996): Euvres complètes. Paris: Gallimard, S. 1551-1620.

Schwab, Martin (1996): Unsichtbares - Sichtbar gemacht. Zu Samuel Becketts $>$ Film<. München: Fink.

Sick, Franziska (2004): »Yvan Golls surreales Filmtheater«. In: Michael Lommel/Isabel Maurer Queipo/Nanette Rißler-Pipka/Volker Roloff (Hg.): Französische Theaterfilme - zwischen Surrealismus und Existentialismus. Bielefeld: transcript, S. 39-64. 